\title{
FARADAY SIGNATURE OF MAGNETIC HELICITY FROM REDUCED DEPOLARIZATION
}

\author{
Axel Brandenburg ${ }^{1,2}$ and Rodion Stepanov ${ }^{3,4}$ \\ ${ }^{1}$ Nordita, KTH Royal Institute of Technology and Stockholm University, Roslagstullsbacken 23, SE-10691 Stockholm, Sweden \\ ${ }^{2}$ Department of Astronomy, AlbaNova University Center, Stockholm University, SE-10691 Stockholm, Sweden \\ ${ }^{3}$ Institute of Continuous Media Mechanics, Korolyov str. 1, 614013 Perm, Russia \\ ${ }^{4}$ Perm National Research Polytechnic University, Komsomolskii Av. 29, 614990 Perm, Russia \\ Received 2014 January 16; accepted 2014 March 18; published 2014 April 21
}

\begin{abstract}
Using one-dimensional models, we show that a helical magnetic field with an appropriate sign of helicity can compensate the Faraday depolarization resulting from the superposition of Faraday-rotated polarization planes from a spatially extended source. For radio emission from a helical magnetic field, the polarization as a function of the square of the wavelength becomes asymmetric with respect to zero. Mathematically speaking, the resulting emission occurs then either at observable or at unobservable (imaginary) wavelengths. We demonstrate that rotation measure (RM) synthesis allows for the reconstruction of the underlying Faraday dispersion function in the former case, but not in the latter. The presence of positive magnetic helicity can thus be detected by observing positive RM in highly polarized regions in the sky and negative RM in weakly polarized regions. Conversely, negative magnetic helicity can be detected by observing negative RM in highly polarized regions and positive RM in weakly polarized regions. The simultaneous presence of two magnetic constituents with opposite signs of helicity is shown to possess signatures that can be quantified through polarization peaks at specific wavelengths and the gradient of the phase of the Faraday dispersion function. Similar polarization peaks can tentatively also be identified for the bi-helical magnetic fields that are generated self-consistently by a dynamo from helically forced turbulence, even though the magnetic energy spectrum is then continuous. Finally, we discuss the possibility of detecting magnetic fields with helical and non-helical properties in external galaxies using the Square Kilometre Array.
\end{abstract}

Key words: galaxies: magnetic fields - methods: data analysis - polarization

Online-only material: color figures

\section{INTRODUCTION}

For many decades, polarized radio emission from external galaxies has been used to infer the strength and structure of their magnetic field. This emission is caused by relativistic electrons gyrating around magnetic field lines and producing the polarized synchrotron emission. The plane of polarization gives an indication about the electric (and thus magnetic) field vectors at the source of emission. The line-of-sight component of the field can be inferred through the Faraday effect that leads to a wavelength-dependent rotation of the plane of polarization. The resulting change of the angle of the polarization plane over a certain wavenumber interval gives the rotation measure (RM), whose variation across different positions within external galaxies gives an idea about the global structure of the magnetic fields of these galaxies (Sofue et al. 1986; Beck et al. 1996, 2005; Fletcher 2010; Beck \& Wielebinski 2013).

In practice, an observer will always see a superposition of different polarization planes from different depths, which can lead to a reduction in the degree of polarization. First, the orientation of the magnetic field changes, causing different polarization planes at different positions. Second, Faraday rotation causes the plane of polarization to rotate. The decrease in polarized emission resulting from this superposition is referred to as Faraday depolarization. This was regarded as a problem that can be alleviated partially by restricting oneself to observations at shorter wavelengths (Soida et al. 2011). This situation has changed with the advent of new generations of radio telescopes that can measure polarized emission over a broad and continuous range of wavelengths. This allows one to apply the method of Burn (1966) that utilizes the wavelength-dependent depolarization to determine the distribution of radio sources with respect to Faraday depth (Brentjens \& de Bruyn 2005; Heald et al. 2009; Gießübel et al. 2013; Frick et al. 2011). However, the interpretation of distributed magnetic fields still remains a challenge (Beck et al. 2012; Bell \& Enßlin 2012).

Of particular interest to the present study is the possibility of detecting helicity of the magnetic field. The helicity of the magnetic field reflects the linkage of the magnetic field (Moffatt 1978). In the context of the large-scale magnetic field in galaxies, one can think of the linkage between the poloidal and toroidal magnetic field components. Three-dimensional visualizations of these two components together, such as Figure 5 of Donner \& Brandenburg (1990), show that the magnetic field lines describe a spiraling pattern. Another manifestation of a helical field is the rotation of a magnetic field vector perpendicular to the line of sight. Determining the presence of such swirling magnetic field patterns would be an important step toward understanding the nature of the underlying dynamo process that is needed to achieve better agreement between observations and theory of astrophysical dynamos. A promising result for probing magnetic helicity in the interstellar medium has been obtained by Volegova \& Stepanov (2010), who have shown that a helical turbulent magnetic field produces a nonzero cross-correlation of RM and the degree of polarization. The sign of the crosscorrelation coefficient permits one to define the sign of the total magnetic helicity. However, the theoretical background of this approach was not clearly understood. Subsequent attempts by Junklewitz \& Enßlin (2011) and Oppermann et al. (2011) did not clarify this effect either, because they excluded the effect of Faraday depolarization from the beginning. To explain the results of Volegova \& Stepanov (2010), we stress the fact that, if the magnetic field is helical, i.e., the magnetic field lines spiral toward or away from the observer, the resulting Faraday 
depolarization can be either enhanced or reduced, depending on the relative signs of magnetic helicity and the line-of-sight component of the magnetic field and thus RM. In a related paper by Horellou \& Fletcher (2014), this effect was used to study the polarized intensity in selected wavelength ranges for both signs of helicity. The exploitation of this effect, which was first discussed by Sokoloff et al. (1998) as an anomalous depolarization due to a twisted magnetic field, is an important motivation behind the present paper.

While the effect of a helical magnetic field is easily understood for simple magnetic spirals, it becomes less obvious in the case of more complicated fields. We are here particularly interested in helical magnetic fields consisting of constituents that have large and small length scales with opposite signs of magnetic helicity. Such fields are called bi-helical and are of central importance in dynamo theory (for a review, see Brandenburg \& Subramanian 2005) and have also been detected in the solar wind (Brandenburg et al. 2011) and on the solar surface (Zhang et al. 2014). There is now also some evidence for helical magnetic fields in the jets emanating from active galactic nuclei (Reichstein \& Gabuzda 2012). We first discuss the observational signatures of singly helical fields and turn then to the case of bi-helical magnetic fields. Next, we discuss a method referred to as cross-correlation analysis using magnetic field configurations similar to those studied in the first part of the paper. Those fields are used to mimic the effects of turbulence consisting of randomly oriented patches with singly helical or bi-helical fields oriented randomly in the sky. Finally, we present preliminary results from more realistic magnetic field configurations generated by a turbulent dynamo in the presence of shear. We conclude with a discussion of the possibilities of detecting helical and bi-helical magnetic fields in external galaxies using the Square Kilometre Array.

\section{COMPENSATING DEPOLARIZATION}

The synchrotron emission of magnetized interstellar or intergalactic media is commonly observed through its total intensity,

$$
I\left(\lambda^{2}\right)=\int_{0}^{\infty} \epsilon(z, \lambda) d z
$$

and through the Stokes $Q$ and $U$ parameters combined into a complex polarization as

$$
P\left(\lambda^{2}\right) \equiv Q+\mathrm{i} U=p_{0} \int_{0}^{\infty} \epsilon(z, \lambda) e^{2 \mathrm{i}\left(\psi(z)+\phi(z) \lambda^{2}\right)} d z
$$

at a given point in the sky. Here $p_{0}$ is the intrinsic polarization (depending on the energy spectrum of the cosmic rays), $\epsilon(z, \lambda) \propto n_{c}(z) B_{\perp}^{\sigma}(z) f(\lambda)$ is the polarized emissivity with $\sigma \approx$ 1.9 being an exponent related to the spectral index (Ginzburg \& Syrovatskii 1965), $n_{c}$ is the cosmic-ray electron density, $B_{\perp}$ is the strength of the magnetic field perpendicular to the line of sight, $f(\lambda) \propto \lambda^{\sigma-1}$ is a wavelength-dependent factor, $\psi(z)$ is the intrinsic polarization angle, $K=0.81 \mathrm{~m}^{-2} \mathrm{~cm}^{3} \mu \mathrm{G}^{-1} \mathrm{pc}^{-1}$ is a constant (Pacholczyk 1970), $\lambda$ is the wavelength,

$$
\phi(z)=-K \int_{0}^{z} n_{e}(s) B_{\|}(s) d s
$$

is the Faraday depth, $n_{e}$ is the electron density (dominated by thermal electrons), $B_{\|}$is the magnetic field along the line of

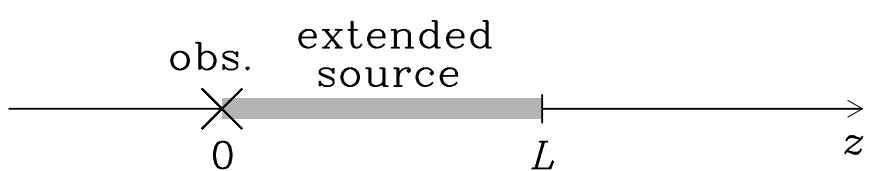

Figure 1. Sketch illustrating position of source and observer.

sight, and $z$ is a coordinate along the line of sight in a Cartesian coordinate system, $(x, y, z)$. Note that Equation (3) implies that the Faraday depth is positive when the mean magnetic field points toward the observer at $z=0$; see Figure 1 and the Appendix for alternative conventions concerning Equations (1)-(3). Variations across the sky are here ignored, so there is no dependence on $x$ and $y$; see Donner \& Brandenburg (1990), Elstner et al. (1992), Brandenburg et al. (1993), and Urbanik et al. (1997) for early applications to mean-field dynamos where this restriction was relaxed. Note that $\epsilon$ also depends on $\lambda$ through a factor $f(\lambda)$, but this term can be moved outside the integral, so it does not constitute a principle problem (Brentjens \& de Bruyn 2005; Bell \& Enßlin 2012), and we shall ignore this complication here. The observed polarization angle is

$$
\chi\left(\lambda^{2}\right)=\frac{1}{2} \arctan (U, Q),
$$

where arctan returns all angles in the range from $-\pi$ to $\pi$, whose tangent yields $U / Q$. It is not to be confused with the intrinsic polarization angle $\psi(z)$.

Since $\boldsymbol{B}$ is assumed independent of $x$ and $y$, the divergencefree condition implies that $B_{\|}=B_{z}=$ const $\equiv B_{\| 0}$. While the assumed independence of $x$ and $y$ may be justified for large-scale fields, it is certainly problematic for small-scale fields. This will be addressed in Section 6. We write the perpendicular magnetic field $\boldsymbol{B}_{\perp}=\left(B_{x}, B_{y}, 0\right)$ in complex form

$$
\mathcal{B}(z) \equiv B_{x}(z)+\mathrm{i} B_{y}(z)=B_{\perp}(z) e^{\mathrm{i} \psi_{B}(z)}
$$

with its phase $\psi_{B}=\arctan \left(B_{y}, B_{x}\right)$. The intrinsic polarization angle $\psi$ is related to $\psi_{B}$ by

$$
\psi=\psi_{B}-\pi / 2 .
$$

Here the $\pi / 2$ term comes from the fact that the plane of polarization is parallel to the electric field and perpendicular to the magnetic field of the radio wave, which, in turn, is parallel to the ambient field $\boldsymbol{B}_{\perp}$. (Note that this term is sometimes omitted; see Waelkens et al. 2009 for such an example. Sokoloff et al. 1998 included it, but dropped the resulting minus sign after their Equation (16).) Due to the factor two in the exponent of Equation (2), which is a consequence of the definition of the Stokes parameters being essentially squared quantities, the phase of the magnetic field has a $\pi$ ambiguity. This is a serious restriction, because it means that the underlying magnetic field cannot be determined fully without additional assumptions.

We now want to determine a condition on the structure of the magnetic field under which the integral in Equation (2) gives maximum contribution, that is, for which the Faraday depolarization is minimal. As was already shown by Sokoloff et al. (1998), this is the case when, for a certain value of $\lambda$, the phase $2\left(\psi(z)+\phi(z) \lambda^{2}\right)$ is a constant. For the purpose of the present discussion we assume constant values of $B_{\perp}, n_{e}$, and $n_{c}$, denoted by $B_{\perp 0}, n_{e 0}$, and $n_{c 0}$, respectively. Therefore, $\phi(z)=-K n_{e 0} B_{\| 0} z$ is linear in $z$, and so the (half) phase under the integral in Equation (2) is given by

$$
\psi(z)+\phi(z) \lambda^{2}=\psi(z)-K n_{e 0} B_{\| 0} \lambda^{2} z,
$$




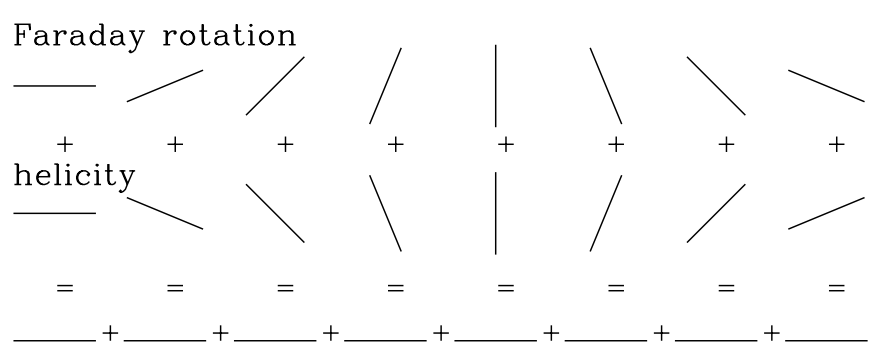

Figure 2. Sketch illustrating the combined effects of Faraday rotation and a helical magnetic field. For a uniform magnetic field, contributions from different depths lead to different angles of the polarization plane. Thus, Faraday rotation alone would lead to Faraday depolarization (sum of the phases of all contributions from the first row), but when $\boldsymbol{B}_{\perp}$ is a helical field rotating properly about the $z$-axis (second row), the contributions from different depths lead to the same observed polarization angle (last row) and Faraday depolarization is thus compensated.

which becomes independent of $z$ and equal to a constant $\psi_{0}$, giving thus maximum contribution to the integral, when

$$
\psi_{B}(z)=\psi_{0}-k z
$$

where $\psi_{0}$ is an arbitrary phase shift and

$$
k=-K n_{e 0} B_{\| 0} \lambda^{2}
$$

is the required wavenumber of the magnetic field. A similar condition was also derived by Arshakian \& Beck (2011), without however explicitly making reference to the helical nature of the magnetic field.

Equation (8) implies that we have a unique solution for the magnetic field that gives maximum contribution to the integral in Equation (2) by essentially canceling the Faraday depolarization from the $\exp \left(2 \mathrm{i} \phi \lambda^{2}\right)$ term, as illustrated in Figure 2. Inserting Equation (8) into Equation (5) and assuming $B_{\perp}=$ const, we have

$$
\boldsymbol{B}=\left(B_{\perp 0} \cos \left(k z-\psi_{0}\right),-B_{\perp 0} \sin \left(k z-\psi_{0}\right), B_{\| 0}\right) .
$$

Such a twisted magnetic field with $\psi_{B}(z) \propto z$ is a Beltrami field and has been considered by Sokoloff et al. (1998) for the demonstration of anomalous depolarization.

As motivated above, we are interested in the magnetic helicity of the field. It is defined as $\langle\boldsymbol{A} \cdot \boldsymbol{B}\rangle$, where angular brackets denote volume averaging and $\boldsymbol{A}$ is the magnetic vector potential with $\boldsymbol{B}=\boldsymbol{\nabla} \times \boldsymbol{A}$ and components $\boldsymbol{A}=\left(B_{x} / k, B_{y} / k+x B_{\| 0}, 0\right)$. Here the linearly varying component $x B_{\| 0}$ is needed to give the constant $B_{\|}=B_{\| 0}$, but this contribution averages out in the calculation of the magnetic helicity,

$$
\langle\boldsymbol{A} \cdot \boldsymbol{B}\rangle=k^{-1} B_{\perp 0}^{2} .
$$

Another quantity of interest, which is based on the current density $\boldsymbol{J}=\boldsymbol{\nabla} \times \boldsymbol{B} / \mu_{0}$ with $\mu_{0}$ being the vacuum permeability, is the current helicity, $\langle\boldsymbol{J} \cdot \boldsymbol{B}\rangle=k B_{\perp 0}^{2} / \mu_{0}$. In the present example, it has the same sign as $\langle\boldsymbol{A} \cdot \boldsymbol{B}\rangle$ and is positive (negative) for positive (negative) values of $k$. Note also that $\psi_{B}$ decreases (increases) with $z$ when the magnetic helicity is positive (negative). Somewhat surprisingly, this implies that the tips of the magnetic field vectors describe a left-handed (righthanded) spiral when magnetic helicity is positive (negative).

For a given magnetic field, that is, prescribed $k$ and $B_{\| 0}$, $\left|P\left(\lambda^{2}\right)\right|$ as a function of $\lambda$ becomes maximal if Equation (9) holds, that is, $\lambda^{2}=-k / K n_{e 0} B_{\| 0}$. Obviously, only $\lambda^{2}>0$ is observable, so only negative (positive) helicities can be detected via the observation of a maximum of $\left|P\left(\lambda^{2}\right)\right|$ if $B_{\| 0}$ is positive (negative), i.e., the field points away from (toward) the observer.

To give an example for typical values of the radio wavelength expected from magnetic fields in the interstellar medium and in external galaxies, let us take $k=2 \pi / \mathrm{kpc}$ for the wavenumber of a field of $1 \mathrm{kpc}$ scale, $n_{e 0}=0.03 \mathrm{~cm}^{-3}$ (Taylor \& Cordes 1993), and $B_{\| 0}=3 \mu \mathrm{G}$; then $\left|P\left(\lambda^{2}\right)\right|$ peaks at $\lambda \approx 30 \mathrm{~cm}$. To probe fields with larger (smaller) length scales, one would need shorter (longer) wavelengths of the radio emission.

\section{FARADAY DISPERSION FUNCTION}

To characterize the observational signature of a helical magnetic field, we compute the corresponding complex polarization as a function of $\lambda^{2}$ using Equation (2). For the purpose of further analysis the polarization can be expressed as a Fourier integral,

$$
P\left(\lambda^{2}\right)=\int_{-\infty}^{\infty} F(\phi) e^{2 \mathrm{i} \phi \lambda^{2}} d \phi
$$

where

$$
F(\phi)=f(\phi) e^{2 \mathrm{i} \psi(\phi)}
$$

is called the Faraday dispersion function (Burn 1966) with $f(\phi)=|F(\phi)|$. Provided that Equation (3) defines a strictly monotonous function $\phi(z)$, we have $d \phi / d z \neq 0$ and can change variables from $z$ to $\phi$ in Equation (2), and we write

$$
f(\phi)=-p_{0} \epsilon(\phi) / K n_{e}(\phi) B_{\|}(\phi),
$$

where the denominator is just $d \phi / d z$ resulting from the transformation from $z$ to $\phi$. The factor two in the exponent of Equation (13) results in the $\pi$ ambiguity. It is therefore useful to characterize signatures of helical magnetic fields directly in terms of $F(\phi)$. This is particularly important, because there is, at least in principle, the chance to reconstruct $F(\phi)$ from $P\left(\lambda^{2}\right)$ using Fourier transformation with respect to the conjugate variable $2 \lambda^{2}$ (Burn 1966). Given the lack of any information about $P\left(\lambda^{2}\right)$ for $\lambda^{2}<0$, we define the synthesized Faraday dispersion function (Burn 1966; Brentjens \& de Bruyn 2005),

$$
F_{\text {syn }}(\phi)=\frac{1}{2 \pi} \int_{0}^{\infty} P\left(\lambda^{2}\right) e^{-2 \mathrm{i} \phi \lambda^{2}} d\left(2 \lambda^{2}\right),
$$

which is supposed to be a reasonable approximation of the actual $F(\phi)$, which would be obtained if the integral in Equation (15) were from $-\infty$ to $\infty$.

We now consider a concrete example using Equation (10) with $k=k_{1}$ to construct a magnetic field in a slab of thickness $L$ with $0 \leqslant z<L$. In the following, we take $\left|k_{1}\right|=2 \pi / L$, i.e., we have within the slab just two nodes in each of the two components of $\boldsymbol{B}_{\perp}$. Outside this range, we assume $\boldsymbol{B}_{\perp}=\mathbf{0}$, but we keep $B_{\|}=B_{\| 0}$ everywhere. The Faraday depth, $\phi=-K n_{e 0} B_{\| 0} z$, is a uniformly varying coordinate, and $\mathcal{R} \equiv \phi(L)=-K n_{e 0} B_{\| 0} L$ is the equivalent intrinsic Faraday RM or simply the Faraday thickness of the slab. Then $\epsilon(\phi) \neq 0$ is the range $0 \leqslant \phi / \mathcal{R} \leqslant 1$. For normalization purposes we introduce here the wavelength $\lambda_{1}$. It is given by

$$
\lambda_{1}^{2}=-k_{1} / K n_{e 0} B_{\| 0}
$$

and determines the peak of the modulus of the resulting complex polarization,

$$
P\left(\lambda^{2}\right)=p_{0} I \hat{P}\left(\mathcal{R}\left(\lambda^{2}-\lambda_{1}^{2}\right)\right)
$$


where

$$
\hat{P}(\xi)=\left(1-e^{2 \mathrm{i} \xi}\right) / 2 \mathrm{i} \xi
$$

is Burn's non-dimensional depolarization function, indicated by a hat. It applies in the absence of magnetic helicity to a uniform slab of Faraday thickness $\mathcal{R}$. Note that in our normalization, $\hat{P}(0)=-1$, where the minus sign is a consequence of the $\pi / 2$ term in Equation (6). Note also that $d \arg (\hat{P}) / d \xi=1$, in spite of the factor two in the exponential function in Equation (18).

The resulting polarization $P\left(\lambda^{2}\right)$ is characterized by two independent parameters of the magnetic field, $k_{1}$ and $B_{\| 0}$, which are represented by $\lambda_{1}^{2}$ and $\mathcal{R}$ in Equation (17). To analyze the form of $P\left(\lambda^{2}\right)$, we consider its modulus and half-phase $\chi\left(\lambda^{2}\right)$ and compare the corresponding functions $F(\phi)$ and $F_{\text {syn }}(\phi)$ for a helical magnetic field with positive helicity $\left(k_{1}>0\right)$ and different signs of $\lambda_{1}^{2}$ (Figure 3 for $\lambda_{1}^{2}>0$ and Figure 4 for $\left.\lambda_{1}^{2}<0\right)$. We see that, as expected, $\left|P\left(\lambda^{2}\right)\right|$ shows a peak at $\lambda^{2}=\lambda_{1}^{2}$, and the sign of $\lambda_{1}^{2}$ depends only on that of the product of $k_{1}$ and $B_{\| 0}$. The polarization angle increases (decreases) with $\lambda^{2}$ for $k_{1}>0$ as shown in Figure 3(b) (Figure 4(b)). This means that the observed RM, RM $=d \chi / d \lambda^{2}$, is positive (negative). Indeed, the case $\lambda_{1}^{2} k_{1}>0$ corresponds to $\mathrm{RM}>0\left(B_{\| 0}<0, \boldsymbol{B}_{\|}\right.$ toward the observer), while $\lambda_{1}^{2} k_{1}<0$ corresponds to $\mathrm{RM}<0$ $\left(B_{\| 0}>0, \boldsymbol{B}_{\|}\right.$points away from the observer).

We note that RM does not depend on $\lambda^{2}$ and that its value is half the Faraday thickness of the slab, i.e., $\mathrm{RM}=\mathcal{R} / 2$. As mentioned above, the reason for the $1 / 2$ factor lies in the mathematical fact that the gradient of the phase of $\hat{P}$ in Equation (18) is 1 and not 2. It is in agreement with the interpretation that for $|F(\phi)|=$ const, $\mathrm{RM}$ is the average value of $\phi$ across the source with $0 \leqslant \phi / \mathcal{R} \leqslant 1$.

Looking at Figures 3(b) and 4(b), we confirm that at the position of the peak at $\lambda^{2}=\lambda_{1}^{2}$ the value of $\chi\left(\lambda^{2}\right)$ is $\pi / 2$. Again, this is a consequence of the $\pi / 2$ term in Equation (6) resulting from the phase shift between magnetic and electric fields of the radio wave and the resulting effect on the plane of polarization. Note also that $\chi\left(\lambda^{2}\right)$ jumps by $\pi / 2$ when $P\left(\lambda^{2}\right)=0$, which is the case when $\lambda^{2}-\lambda_{1}^{2}$ is a non-vanishing half-integer multiple of $\left|\lambda_{1}^{2}\right|$. Unlike the jump at $\lambda^{2}=\lambda_{1}^{2}$ by $\pi$ because of $\pi$ ambiguity, the $\pi / 2$ jumps are physical singularities in the polarization angle as a function of $\lambda^{2}$. These $\pi / 2$ discontinuities were also noted by Burn (1966) and are a natural consequence of decomposing a complex function with zeros such as Equation (18) into modulus and phase.

Since the product of $k_{1}$ and RM is positive in Figure 3, polarized emission occurs now in the range $0<\lambda^{2}<\infty$ and would therefore be observable. As expected, the synthesized $F_{\text {syn }}(\phi)$ agrees therefore fairly well with the original $F(\phi)$; compare the black with the red dashed lines in Figure 3. Real and imaginary parts of $F(\phi)$ and $F_{\text {syn }}(\phi)$ are phase-shifted by $\pi / 2$ relative to each other, which is indicative of a helical field; see Equation (10). Note also that $|F(\phi)|$ is constant and $\psi(\phi)$ is decreasing with increasing $\phi$, as seen from Equation (8). Again, the agreement between $F(\phi)$ and $F_{\text {syn }}(\phi)$ is rather good.

If $k_{1} \mathrm{RM}<0$, the peak occurs at negative values of $\lambda^{2}$ and is thus unobservable. In that case, there would be essentially no polarized emission and the RM-synthesized Faraday dispersion function is very poor; see Figures 4(c)-(e). A quantitative analysis of the reconstruction of the Faraday dispersion function for different wavelength ranges and radio telescopes is given by Horellou \& Fletcher (2014). The width of the polarization peaks depends on $\mathcal{R}$. It is sharper for a thicker emitting
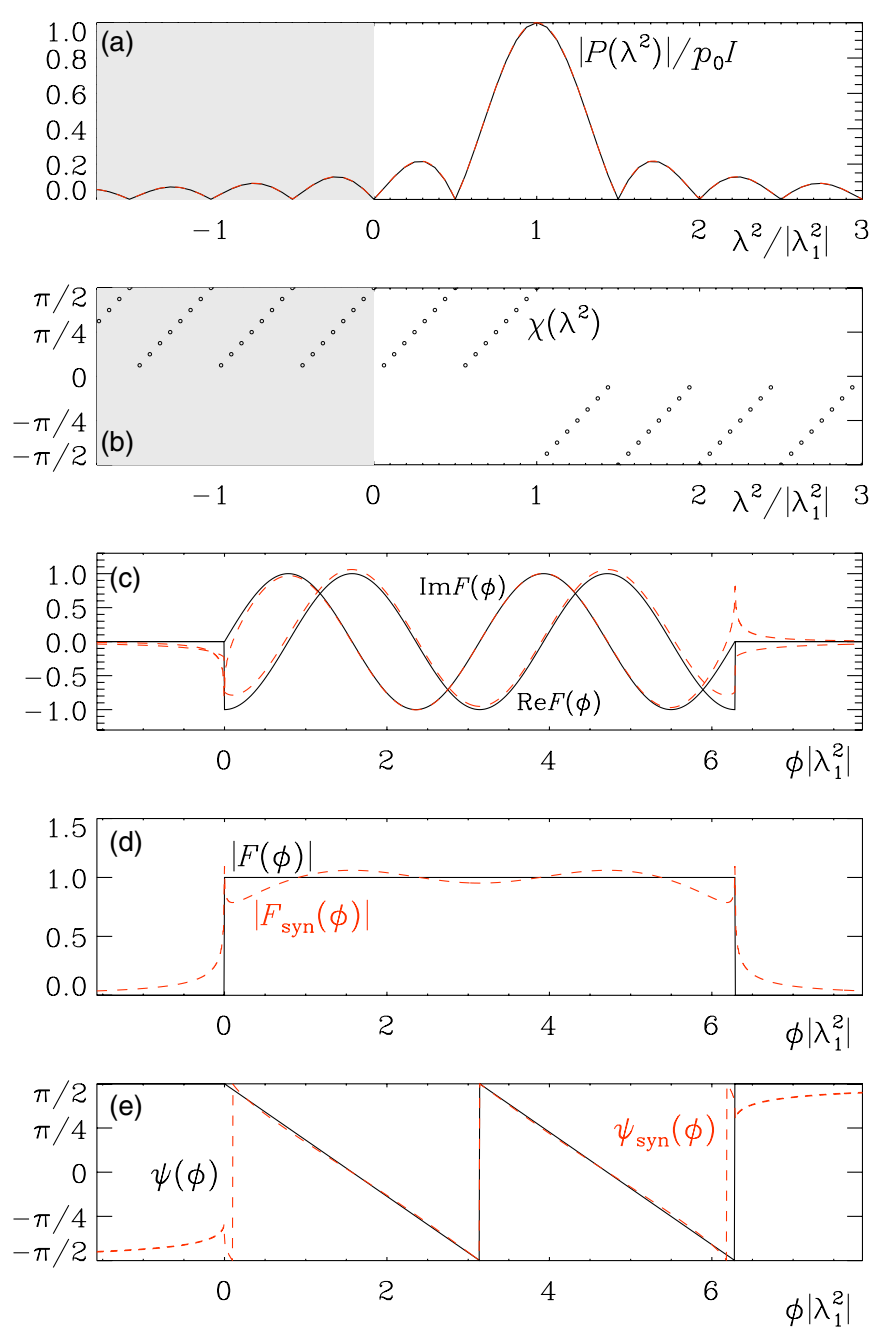

Figure 3. (a) $\left|P\left(\lambda^{2}\right)\right|$, (b) $\chi\left(\lambda^{2}\right)=\arg (P) / 2$, (c) real and imaginary parts of $F(\phi),(\mathrm{d})|F(\phi)|$, and (e) $\psi(\phi)$ for a magnetic field with positive helicity $k_{1}>0$ and positive $\lambda_{1}^{2}>0$. In panels (a) and (b), the unobservable range $\lambda^{2}<0$ is marked in gray. In panels (c)-(e), the quantities for the synthesized Faraday dispersion function are overplotted as red dashed lines.

(A color version of this figure is available in the online journal.)

region and broader for a thinner one. In the limit of an infinitely thick slab, $P\left(\lambda^{2}\right)$ becomes a $\delta$ function with no side lobes, so the remaining discrepancy between $F(\phi)$ and $F_{\text {syn }}(\phi)$ in Figures 3(c)-(e) would disappear. Perfect reconstruction of a non-helical magnetic field in a slab can be achieved only with additional assumptions about the symmetry of the source (Frick et al. 2010).

\section{BI-HELICAL MAGNETIC FIELDS}

In galaxies, magnetic fields are thought to be produced and maintained by a turbulent dynamo involving a so-called $\alpha$ effect. This leads to helical large-scale magnetic fields (e.g., Moffatt 1978). However, since magnetic helicity is an invariant in ideal magnetohydrodynamics (Woltjer 1958), no net magnetic helicity can be produced. Instead, a bi-helical magnetic field is generated, which has an additional small-scale constituent of opposite magnetic helicity. This is an idealized situation, because in reality there will be magnetic helicity fluxes (Kleeorin et al. 2000) that influence the local helicity balance. Nevertheless, to study this idealized case in more detail, we consider as a simple example the following one-dimensional, 

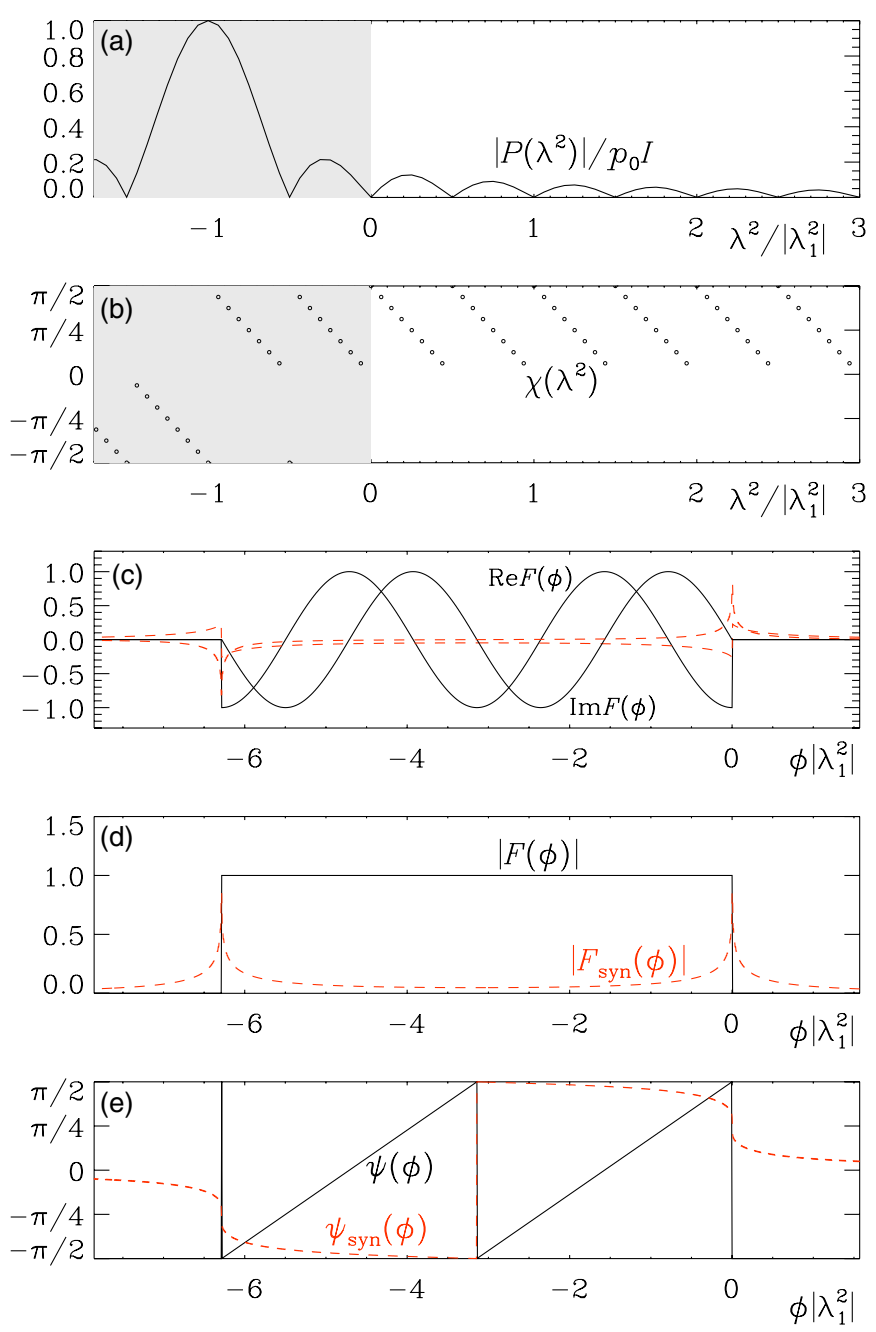

Figure 4. Same as Figure 3, but for $\lambda_{1}^{2}<0$, keeping however $k_{1}>0$. (A color version of this figure is available in the online journal.)

bi-helical magnetic field:

$$
\boldsymbol{B}=\left(\begin{array}{c}
B_{1} \cos k_{1} z+B_{2} \cos \left(k_{2} z+\varphi\right) \\
-B_{1} \sin k_{1} z-B_{2} \sin \left(k_{2} z+\varphi\right) \\
B_{\| 0}
\end{array}\right),
$$

where $k_{1}$ is the wavenumber of the constituent with amplitude $B_{1}, k_{2}$ is that of the constituent with amplitude $B_{2}$, and $\varphi$ is an arbitrary phase shift between the two constituents. The magnetic and current helicities of the total field are respectively given by

$$
\langle\boldsymbol{A} \cdot \boldsymbol{B}\rangle=k_{1}^{-1} B_{1}^{2}+k_{2}^{-1} B_{2}^{2}, \quad \mu_{0}\langle\boldsymbol{J} \cdot \boldsymbol{B}\rangle=k_{1} B_{1}^{2}+k_{2} B_{2}^{2} .
$$

Thus, the field has zero magnetic helicity when $-k_{2} / k_{1}=$ $B_{2}^{2} / B_{1}^{2}$ and zero current helicity when $B_{2}^{2} / B_{1}^{2}$ is $-k_{1} / k_{2}$, which is just the inverse scale ratio. The latter situation is realized in a periodic domain after a resistive timescale (Brandenburg 2001), while the former is expected to hold on short timescales (Field \& Blackman 2002; Blackman \& Brandenburg 2002). As alluded to above, in reality there are magnetic helicity fluxes. In practice, they tend to lead to a situation that is between these two extreme cases (Brandenburg et al. 2009).

We emphasize that the sign of $k_{i}$ (with $i=1$ or 2) determines also the sign of the helicity of the corresponding field constituent. In the following we take $k_{1}>0$ and $k_{2}<0$ with $\left|k_{2}\right|>k_{1}$, so the field with amplitude $B_{1}$ is a large-scale field with positive helicity, and that with amplitude $B_{2}$ is a small-scale one with negative helicity. This is also the situation expected to be applicable to the upper disk plane of galaxies, i.e., where the angular velocity vector points in the opposite direction as gravity.

We vary $k_{1}$ and $k_{2}$ to identify features in the results for $P\left(\lambda^{2}\right)$ and $F(\phi)$ that can be related to these wavenumbers. We define corresponding wavenumbers in Faraday space

$$
\lambda_{i}^{2}=-k_{i} / K n_{e 0} B_{\| 0},
$$

which we use to define the two quantities

$$
\lambda_{p}^{2}=\left(\lambda_{1}^{2}+\lambda_{2}^{2}\right) / 2 \text { and } \Delta \lambda^{2}=\left(\lambda_{1}^{2}-\lambda_{2}^{2}\right) / 2 .
$$

Note that, even though each of the two constituents of the bihelical field has a constant modulus, the modulus of the sum is not constant. Instead, it is seen from the example shown in Figure 6 that it varies periodically like

$$
|\hat{\mathcal{B}}|^{2} \sim \cos \left(2 \phi \Delta \lambda^{2}-\varphi\right) .
$$

Under the assumption that the exponent of the polarized emissivity is $\sigma=2$, an analytic solution Equation (2) can be given in terms of Burn's depolarization function (18) as

$$
\begin{aligned}
P\left(\lambda^{2}\right) / p_{0} I= & \epsilon_{1} \hat{P}\left(\mathcal{R}\left(\lambda^{2}-\lambda_{1}^{2}\right)\right) \\
& +\epsilon_{2} \hat{P}\left(\mathcal{R}\left(\lambda^{2}-\lambda_{2}^{2}\right)\right) \\
& +\epsilon_{p} \hat{P}\left(\mathcal{R}\left(\lambda^{2}-\lambda_{p}^{2}\right)\right)
\end{aligned}
$$

where $\epsilon_{1}=B_{1}^{2} / B_{*}^{2}, \epsilon_{2}=B_{2}^{2} / B_{*}^{2}$, and $\epsilon_{p}=2 B_{1} B_{2} / B_{*}^{2}$, with $B_{*}^{2}=B_{1}^{2}+B_{2}^{2}+2 B_{1} B_{2} \operatorname{sinc}\left(2 \Delta \lambda^{2}\right)$, are normalization constants. There are three peaks of $P\left(\lambda^{2}\right)$ : two peaks are located at $\lambda_{1}^{2}$ and $\lambda_{2}^{2}$, and a third one appears at $\lambda_{p}^{2}$. They are shown in Figure 5 for the case $B_{2} / B_{1}=1$. As is clear from Equation (24), the separation between adjacent peaks is given by $\left|\Delta \lambda^{2}\right|$. This solution is independent of the phase shift $\varphi$ between the two constituents.

To understand the signatures of a bi-helical magnetic field in the Faraday dispersion function, let us recall that the wavenumbers of each of the two constituents contribute to the gradient $d \psi / d \phi$. It is therefore plausible that in the case $B_{1}=B_{2}$ the result is just the average of the two, i.e.,

$$
d \psi / d \phi=-\lambda_{p}^{2} .
$$

This property of $d \psi / d \phi$ is preserved regardless of the $\pi$ ambiguity. To demonstrate this, we compare in Figure 6 both $\psi_{B} \equiv \arctan \left(B_{y}, B_{x}\right)$ (all angles in the range from $-\pi$ to $\pi$ that yield $\left.B_{y} / B_{x}\right)$ and $\psi_{B}^{\prime}=\arctan \left(B_{y} / B_{x}\right)$, which is confined to the range from $-\pi / 2$ to $\pi / 2$. As stated in Section $2, d \psi_{B} / d \phi$ is negative when the product $k B_{\| 0}$ is positive. This is indeed in agreement with Figure 6.

Interestingly, $\psi_{B}^{\prime}$ is simpler than $\psi_{B}$ in that the former has no phase jumps other than those required for $\psi_{B}^{\prime}$ to remain in the range from $-\pi / 2$ to $\pi / 2$. By contrast, $\psi_{B}$ shows phase jumps by $\pi$ at all locations where $|\mathcal{B}|$ vanishes; compare Figures 6(a) and (b). Ignoring these phase jumps, i.e., reconstructing the field from $|\mathcal{B}|$ and $\psi_{B}^{\prime}$, instead of $\psi_{B}$, would render the underlying magnetic field discontinuous.

Our statements can be confirmed by evaluating Equation (24) or by computing numerically examples for different combinations of $k_{1}$ and $k_{2}$; see also Figure 5. Thus, we can summarize 

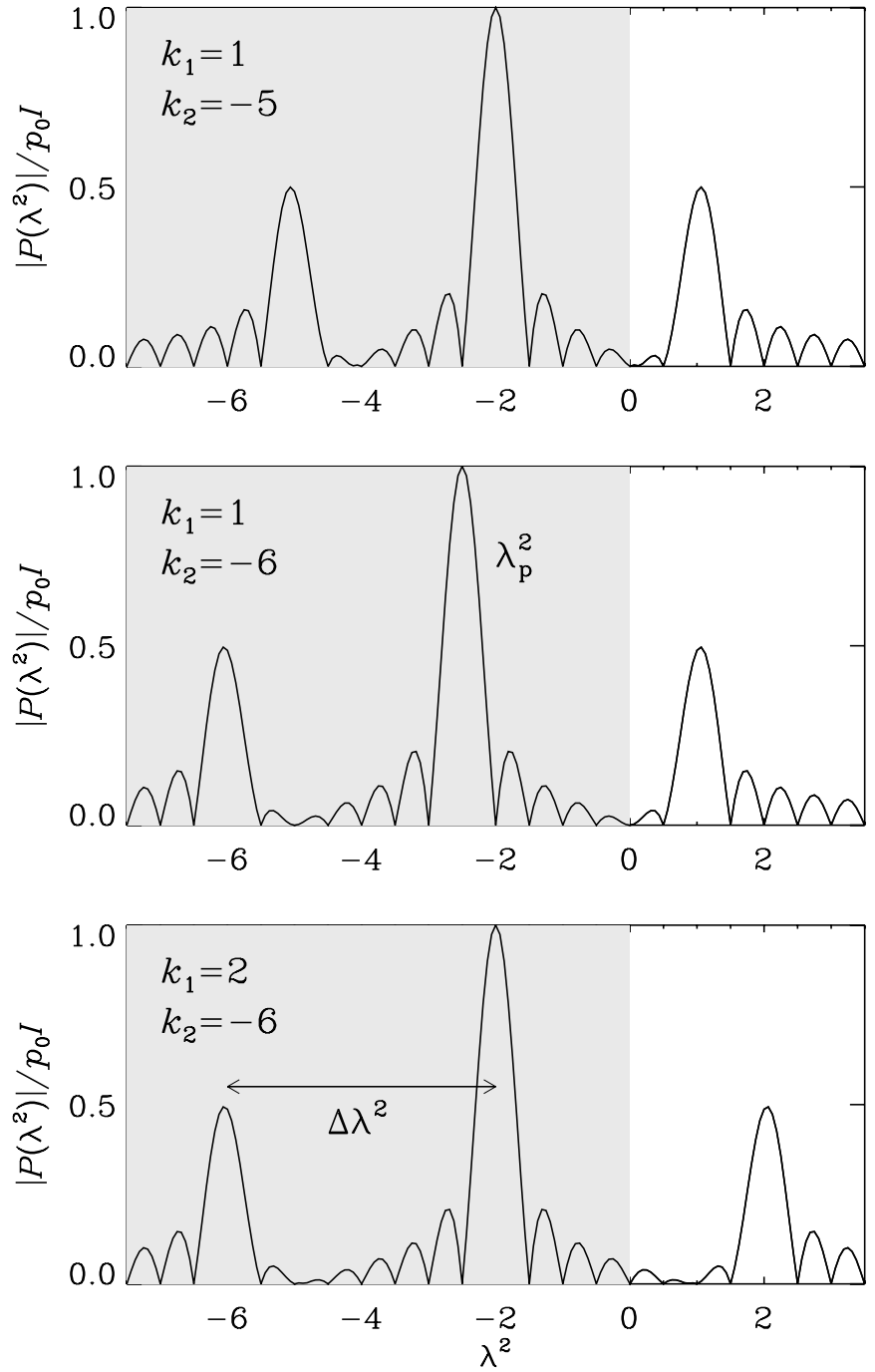

Figure 5. $\left|P\left(\lambda^{2}\right)\right|$ for different values of $k_{1}$ and $k_{2}$ and $B_{2} / B_{1}=1$ using $\mathrm{RM}>0$. The unobservable range $\lambda^{2}<0$ is marked in gray.

that a bi-helical magnetic field with wavenumbers $k_{1}$ and $k_{2}$ results in a clear signature in the Faraday dispersion function in that the frequency of its modulus is given by $2 \Delta \lambda^{2}$ (Figure 6(a)), while indeed $d \psi / d \phi=-\lambda_{p}^{2}$ (Figure 6(b)).

To appreciate the features of a bi-helical magnetic field in the complex polarization $P$, let us note that a Fourier transformation of the complex function $\mathcal{B}$, defined in Equation (5) and now applied to the bi-helical field defined in Equation (19), would produce peaks at wavenumbers $k_{1}$ and $k_{2}$. However, in the Fourier transformation defined through Equation (12), wavenumbers correspond to the Fourier variable $2 \lambda^{2}$. Thus, if the Faraday dispersion function was given by $\mathcal{B}(\phi)$, the corresponding Fourier transform $\hat{\mathcal{B}}\left(2 \lambda^{2}\right)$ shows peaks at $2 \lambda^{2} / \lambda_{1}^{2}=1$ and $k_{2} / k_{1}=-5$; see Figure $6(\mathrm{c})$. In reality, the Faraday dispersion function is given by $\mathcal{B}^{2}$ (assuming here $\sigma=2$ ). A Fourier transformation of such a squared function has a peak at $k_{1}+k_{2}$ and side lobes at $k_{1}+k_{2} \pm\left|k_{1}-k_{2}\right|=2 k_{1}$ or $2 k_{2}$. Thus, the corresponding Fourier transform, which we can now call $P\left(2 \lambda^{2}\right)$, has peaks at $2 \lambda^{2} / \lambda_{1}^{2}=2$ and $2 k_{2} / k_{1}=-10$, together with a larger one in between; see Figure 6(d).

The above considerations assume that the amplitudes of the two constituents are approximately equal. When $B_{2} / B_{1}$ is either very small or very large, the type of the resulting polarization
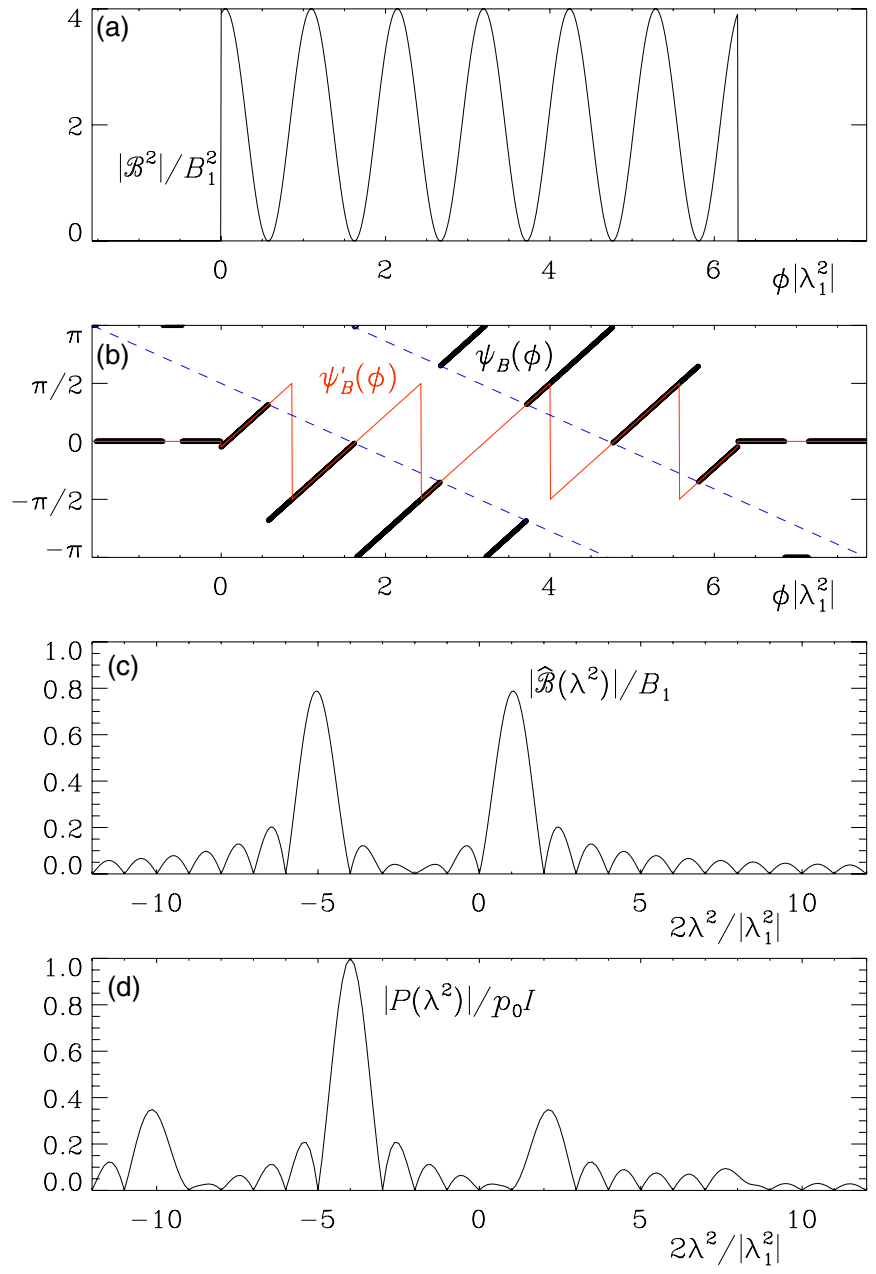

Figure 6. (a) $|B|^{2}(\phi)$, (b) $\psi_{B}(\phi)$ and $\psi_{B}^{\prime}(\phi)$, (c) $\hat{\mathcal{B}}\left(2 \lambda^{2}\right)$, and (d) $P\left(2 \lambda^{2}\right)$ for a bi-helical magnetic field with $k_{2} / k_{1}=-5$ using RM $>0$. In panel (b), the dashed blue lines correspond to $\pi / 2-\phi\left|\lambda_{1}^{2}\right|$ and $3 \pi / 2-\phi\left|\lambda_{1}^{2}\right|$ and mark the points where the phase of $\psi_{B}(\phi)$ jumps.

(A color version of this figure is available in the online journal.)

signal will be determined by the dominating one of the two constituents. Figure 7 confirms that the peak at $\lambda^{2}=\lambda_{p}^{2}$ diminishes when $B_{2} / B_{1}$ becomes either much larger than unity or much smaller than unity. Not surprisingly, a peak at $\lambda^{2}=\lambda_{2}^{2}$ begins to emerge when $B_{2}$ becomes large (bottom panels of Figure 7), and one at $\lambda^{2}=\lambda_{1}^{2}$ emerges when $B_{1}$ becomes large (top panels of Figure 7). In the latter case, however, most of the polarized emission occurs formally for $\lambda^{2}<0$.

Figure 7 suggests that two of the peaks have a similar height when $\langle\boldsymbol{J} \cdot \boldsymbol{B}\rangle=0$ (second row of Figure 7 ) or when $\langle\boldsymbol{A} \cdot \boldsymbol{B}\rangle=0$ (fourth row of Figure 7). While this is not a general result, there is, however, a tendency for those two peaks to survive even in the limits of very large or very small ratios of $\left|k_{1} / k_{2}\right|$.

Our considerations of helical and bi-helical magnetic fields have shown that the distributions of $P\left(\lambda^{2}\right)$ are asymmetric with respect to $\lambda=0$. This underlines again that the reconstruction of missing data for negative values of $\lambda^{2}$ from symmetry arguments, e.g., that $P\left(-\lambda^{2}\right)=P^{*}\left(\lambda^{2}\right)$, would be impossible when the magnetic field is helical and the helicity is of unsuitable sign (i.e., $k_{1} \mathrm{RM}<0$ ) for a given sign of RM. This is because the phase of the Faraday dispersion function shows then significant dependence on Faraday depth, so the term $\psi(z)$ cannot be 

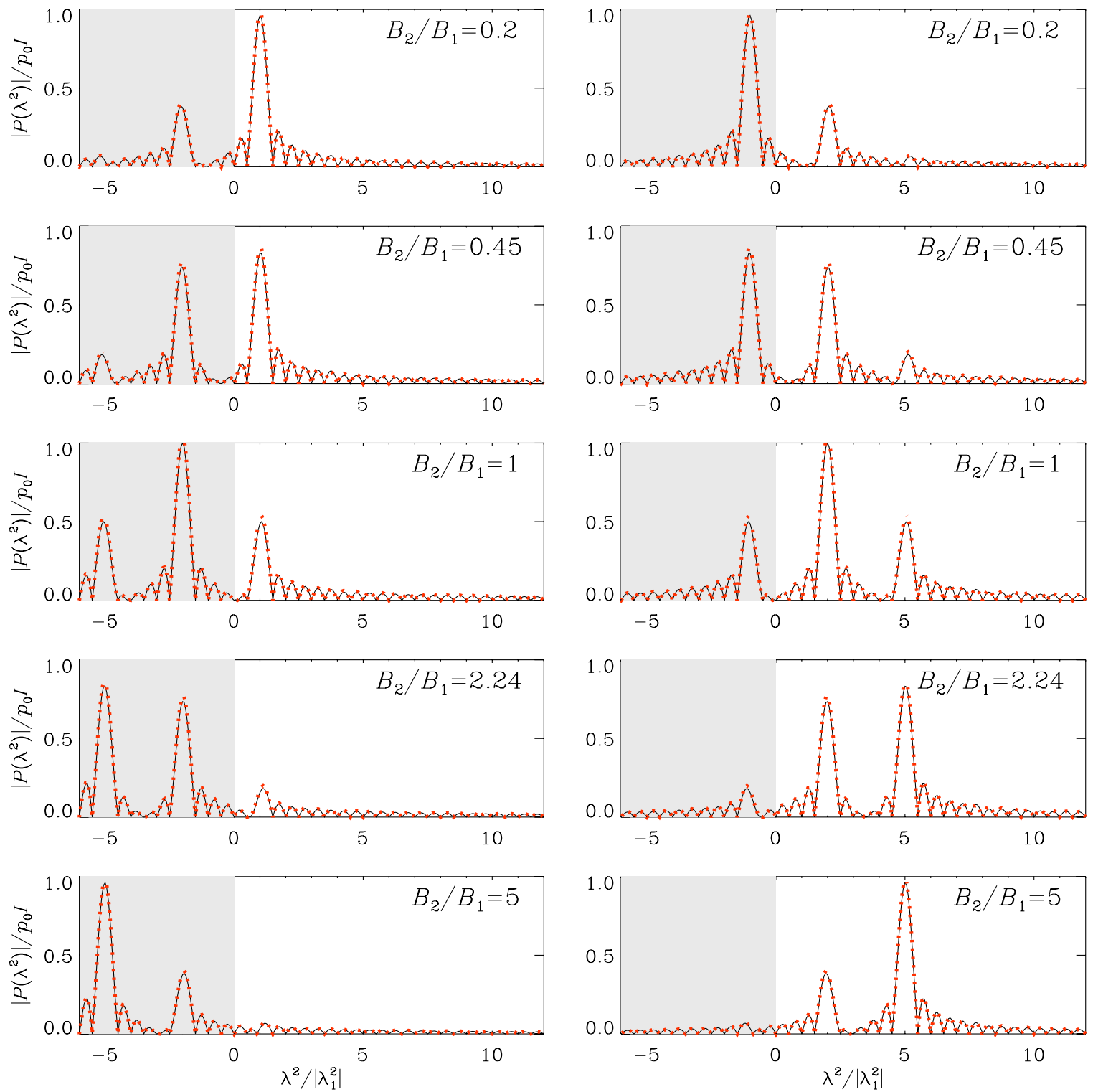

Figure 7. Dependence of $\left|P\left(\lambda^{2}\right)\right|$ for different values of $B_{1} / B_{2}$ and $k_{2} / k_{1}=-5$ using RM $>0$ (left column) and RM $<0$ (right column). The region with $\lambda^{2}<0$ is marked in gray. The analytic solutions with $\sigma=2$ are shown as red dotted lines, while the numerical one for $\sigma=1.9$ is shown as a black solid line. For $B_{2} / B_{1}=0.45$ in the second row we have $\langle\boldsymbol{J} \cdot \boldsymbol{B}\rangle=0$, while for 2.24 in the fourth row we have $\langle\boldsymbol{A} \cdot \boldsymbol{B}\rangle=0$.

(A color version of this figure is available in the online journal.)

pulled outside the integral of Equation (2), which is a critical assumption often made in this connection (Burn 1966).

It is remarkable that in all cases with helical magnetic fields, there is a particular value $\lambda^{2}$ for which the polarization approaches the maximum value of $|P| / p_{0} I=1$. Depending on the relative strengths of $B_{1}$ and $B_{2}$, this peak can be either at $\lambda^{2}=\lambda_{1}^{2}, \lambda_{2}^{2}$, or at $\lambda_{p}^{2}=\left(\lambda_{1}^{2}+\lambda_{2}^{2}\right) / 2$; see Figure 7 and Equation (24).

\section{CROSS-CORRELATION ANALYSIS OF $|P|$ VERSUS RM}

Our present investigations have implications that help understand earlier work in the field. Recent surveys of polarized emission in the interstellar medium have provided continuous distributions of $Q$ and $U$ on the sky for certain ranges of wavelengths. Due to finite beam size, only a small number of independent lines of sight are available for analysis. Probing mag- netic helicity with a cross-correlation analysis between RM and the polarization degree $\mathcal{P} \equiv|P| / p_{0} I$ had been suggested by Volegova \& Stepanov (2010) using simulated data. While the numerical demonstration of the method was convincing, no theoretical proof or explanation had been available yet.

To study this idea further, we imagine turbulence being approximated by a set of cells possessing locally a homogeneous helical magnetic field as in Equation (10). The dominating scale of the turbulence can be attributed to the size of the cells. The direction of each helix is taken to be random, but for a large number of cells there are always some for which it is almost parallel to the line of sight (top right panel of Figure 8). Only such cells are considered in the following. In Volegova \& Stepanov (2010), the cross-correlation coefficient between synthetic maps of RM and the polarization degree $\mathcal{P}$ was found to be positive (negative) when the total magnetic helicity in the domain was prevailingly positive (negative). Since the direction of $B_{\|}$is random, the average value of RM over all cells is 


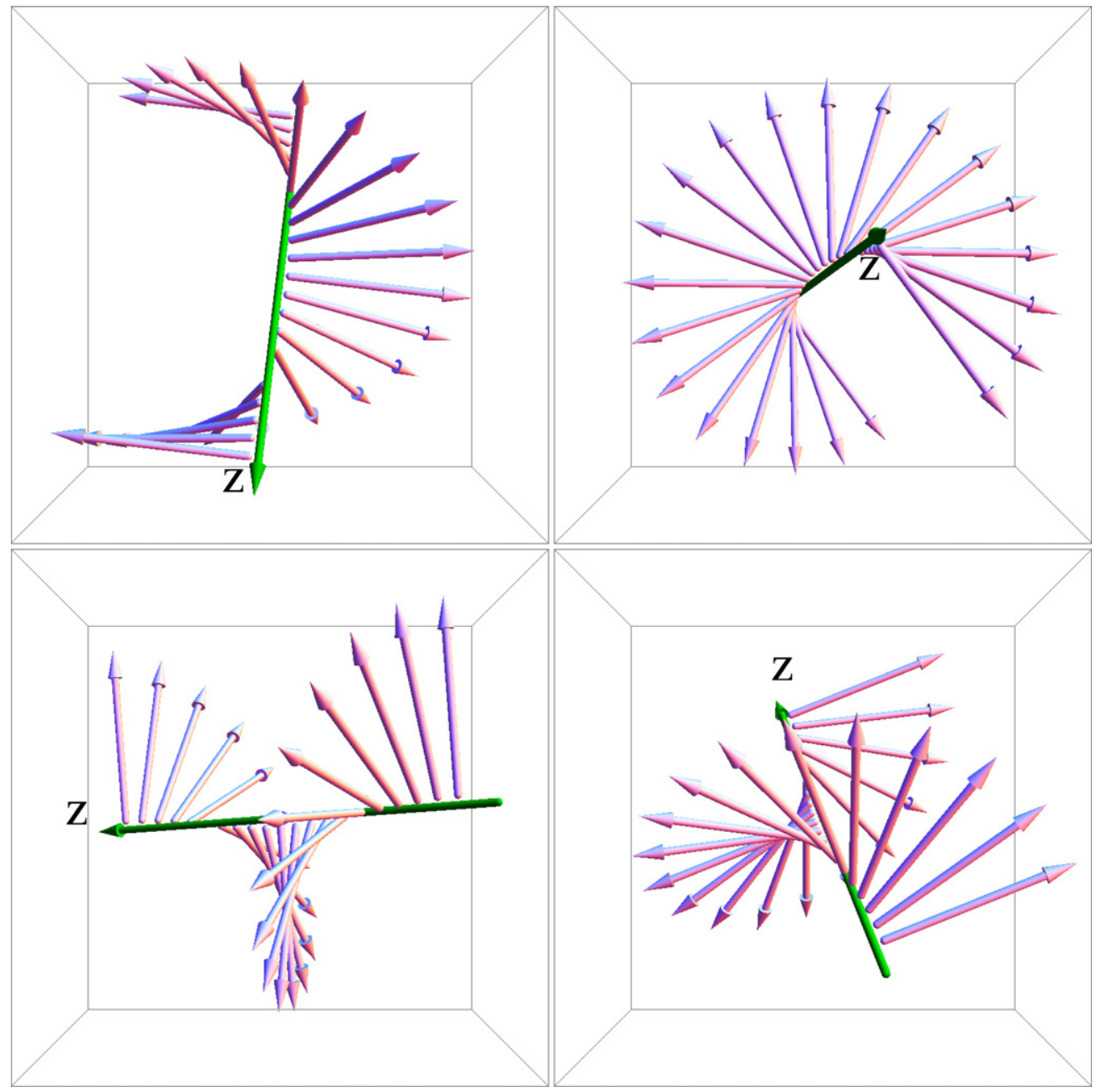

Figure 8. Set of cells each with a singly helical magnetic field of positive helicity. The tips of the vectors describe a left-handed spiral.

(A color version of this figure is available in the online journal.)

zero. Then the cross-correlation coefficient is determined by the average value of the product $\mathrm{RM} \mathcal{P}$, which can be considered as a weighted average of RM with the weight $\mathcal{P}$. Having in mind Equation (11), we recall that the maximum polarization corresponds to cells with positive helicity and positive RM or, alternatively, negative helicity and negative RM. Minimum polarization comes from cells with opposite sign of helicity and RM. Thus, if the number of cells with positive and negative helicity is about the same, then positive and negative RMs are weighted equally and the cross-correlation is zero. If the cells with positive (negative) helicity are dominant, then $\langle\mathrm{RM} \mathcal{P}\rangle$ is positive (negative).

In the following, another test is suggested for the crosscorrelation diagnostics. We consider the averaged polarization $\left\langle|P| / p_{0} I\right\rangle$ by averaging over $\lambda^{2}$, using, however, only one cell. In Figure 9 we show first the dependence of $\left\langle|P| / p_{0} I\right\rangle$ on RM for different wavenumbers using a singly helical magnetic field. Here we have averaged over wavelengths in the range $0<\lambda^{2} \leqslant \lambda_{1}^{2}$. We see that, for positive (negative) helicities, the averaged polarization is largest for positive (negative) values of RM.

Next, in Figure 10 we show correlation plots using data from Figure 7 for the case of a bi-helical field, where we take the average value of $\left|P\left(\lambda^{2}\right)\right| / p_{0} I$ for $0<\lambda^{2} / \lambda_{1}^{2} \leqslant 10$. We also compute the corresponding results for $1 / 2$ and $1 / 10$ of the reference value of $\mathrm{RM}$, namely, $\mathrm{RM} / \mathrm{RM}_{0}=1,0.5$, and 0.1 ,
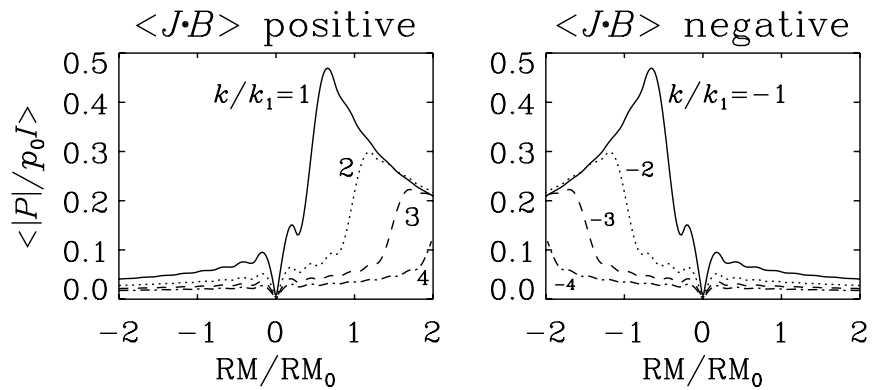

Figure 9. Dependence of $\left\langle P / p_{0} I\right\rangle$ on RM for different wavenumbers $k$ (relative to a reference wavenumber $k_{1}$ ) and cases with positive and negative current helicities (positive and negative values of $k$ ) using an average over $0<\lambda^{2} / \lambda_{1}^{2} \leqslant 10$.

where $\mathrm{RM}_{0} \lambda_{1}^{2}=\pi$. In the cases shown in Figure 7, the current helicity $\langle\boldsymbol{J} \cdot \boldsymbol{B}\rangle$ is negative, so the resulting polarized emission is small for positive values of RM, but large for negative values of RM. This results in a negative correlation (see right-hand panel of Figure 10), as expected from the analysis of Volegova \& Stepanov (2010). Conversely, when we change the signs of $k_{1}$ and $k_{2}$, which corresponds to positive current helicity, the correlation is positive. Thus, our present results support the findings of Volegova \& Stepanov (2010) at a qualitative level and demonstrate, furthermore, that for bi-helical magnetic 

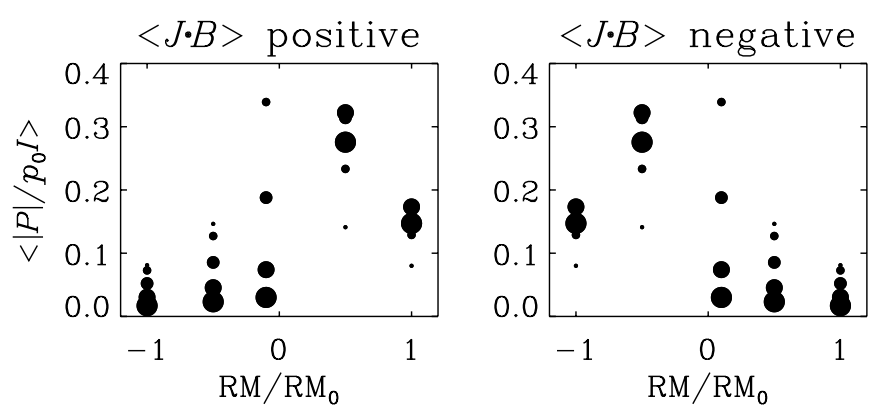

Figure 10. Correlation between RM and $\left\langle P / p_{0} I\right\rangle$ for cases with positive and negative current helicities. The size of the symbols reflects the value of $B_{2} / B_{1}$ in Figure 7.

fields their method is more sensitive to current helicity than to magnetic helicity, which has the opposite sign in the example considered in Figure 10.

\section{TURBULENCE-GENERATED MAGNETIC FIELDS}

In this paper we have analyzed an extremely simple model of astrophysical magnetic fields. One potential problem is the fact that the actual magnetic field possesses not just two scales, but there is a continuous spectrum of scales. The other problem is that the line-of-sight magnetic field is not constant, so $\phi(z)$ becomes nonlinear and is different for each line of sight. To assess how the results from our idealized models are affected by these issues, we now analyze a snapshot from a turbulence simulation exhibiting large-scale dynamo action.

In our model, turbulence is driven through helical forcing, as was also done in Brandenburg (2001), where the forcing acts only in a narrow band of wavenumbers with an average value $k_{\mathrm{f}}$ that is five times larger than the smallest wavenumber that fits into the computational domain, $\left|k_{1}\right|$. Thus, $k_{2} /\left|k_{1}\right|=5$. The resulting kinetic energy spectrum is, however, continuous for $k>k_{\mathrm{f}}$ and extends until the dissipative cutoff wavenumber, whose value depends on the Reynolds number; see Figure 1(b) of Brandenburg et al. (2012) for a higher resolution simulation. To model the effects of a significant line-of-sight magnetic field in a physically meaningful way, we include shear. Our model is thus similar to that of Käpylä \& Brandenburg (2009), where dynamo waves are found to travel in the span-wise direction. The boundary conditions are (shearing) periodic, and the kinetic helicity has the same sign throughout the computational domain, so there is no equator in this model.

Our simulation has been carried out using the PENCIL CODE ${ }^{5}$ with a resolution of $192^{3}$ mesh points and is characterized by the magnetic Reynolds and Prandtl numbers, $R_{\mathrm{m}} \equiv u_{\mathrm{rms}} / \eta k_{\mathrm{f}}=120$ and $\operatorname{Pr}_{M} \equiv v / \eta=1$, respectively, as well as the shear parameter $\mathrm{Sh}=S / u_{\mathrm{rms}} k_{\mathrm{f}}=0.16$. Here $u_{\mathrm{rms}}$ is the rms velocity of the turbulence, $\eta$ is the magnetic diffusivity, $v$ is the kinematic viscosity, and $S=|\nabla \overline{\boldsymbol{U}}|$ is the shear rate of the mean flow $\overline{\boldsymbol{U}}$.

It turns out that the nonlinearity of $\phi(z)$ is a much more serious problem than the existence of a continuous spectrum of scales. To demonstrate this, we begin with the best-case scenario assuming $B_{\|}=$const, so $\phi(z)$ is linear in $z$. As in Figure 6, we consider first the complex variable $\mathcal{B}$, which characterizes the perpendicular magnetic field component in the projected plane of the sky; see left column of Figure 11. Its Fourier transform along the line of sight, $\hat{\mathcal{B}}\left(2 \lambda^{2}\right)$, averaged over all points in the plane, shows clearly the small-scale magnetic field with positive helicity at $2 \lambda^{2} /\left|\lambda_{1}^{2}\right|=+5$ and the large-scale magnetic field with negative magnetic helicity at $2 \lambda^{2} /\left|\lambda_{1}^{2}\right|=-1$, corresponding to the lowest wavenumber of the domain. For $B_{\|}=$const and $\sigma=2$, we can compute $\left|P\left(2 \lambda^{2}\right)\right|$ as the Fourier transform of $\mathcal{B}^{2}$. Its average over all points in the plane shows peaks at $2 \lambda^{2} /\left|\lambda_{1}^{2}\right|=-3$ (which is slightly lower than the expected value -2 ) and at +9 (which is slightly below the expected value of +10 ). Thus, we may tentatively conclude that the presence of a continuous spectrum of scales in the magnetic field has a less serious effect on the polarization peaks than the nonlinearity of $\phi$ that will be discussed next. There is, however, a peak at $\lambda^{2}=0$, which we have not seen in the two-scale model. A more detailed inspection shows that the overall depolarization is generally rather strong when the field is turbulent. This weakens the compensation of depolarization by helicity (Section 2), leaving behind the finite polarization at $\lambda^{2}=0$ due to the contribution of a mean $\boldsymbol{B}_{\perp}$ along the line of sight. We have verified that the removal of a mean $\boldsymbol{B}_{\perp}$ by replacing $\boldsymbol{B}_{\perp} \rightarrow \boldsymbol{B}_{\perp}-\left\langle\boldsymbol{B}_{\perp}\right\rangle_{\|}$can reduce the peak at $\lambda^{2}=0$ in most cases.
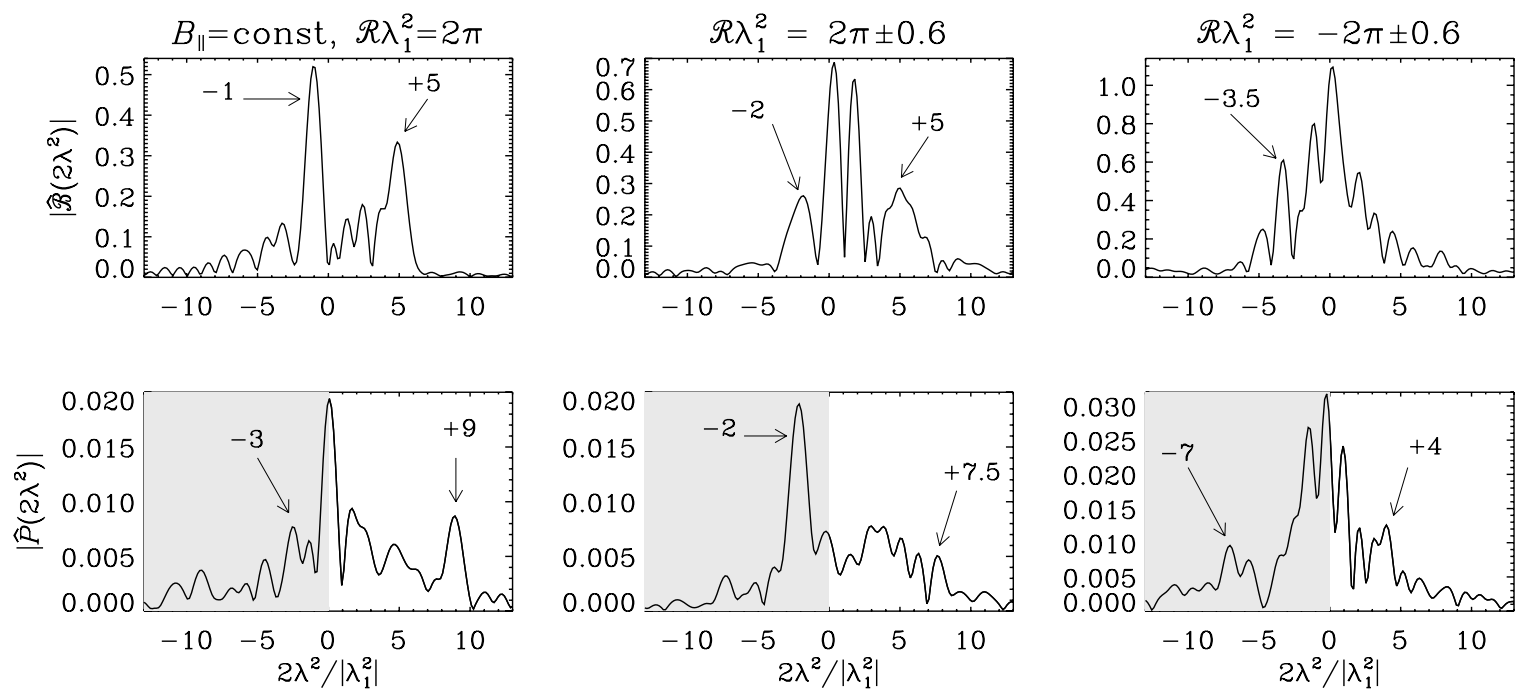

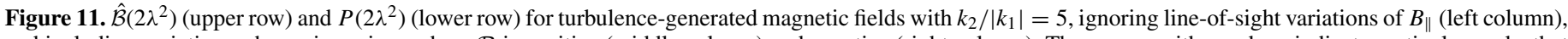

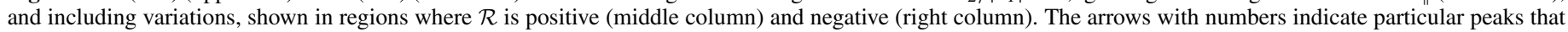
are discussed in the text. 


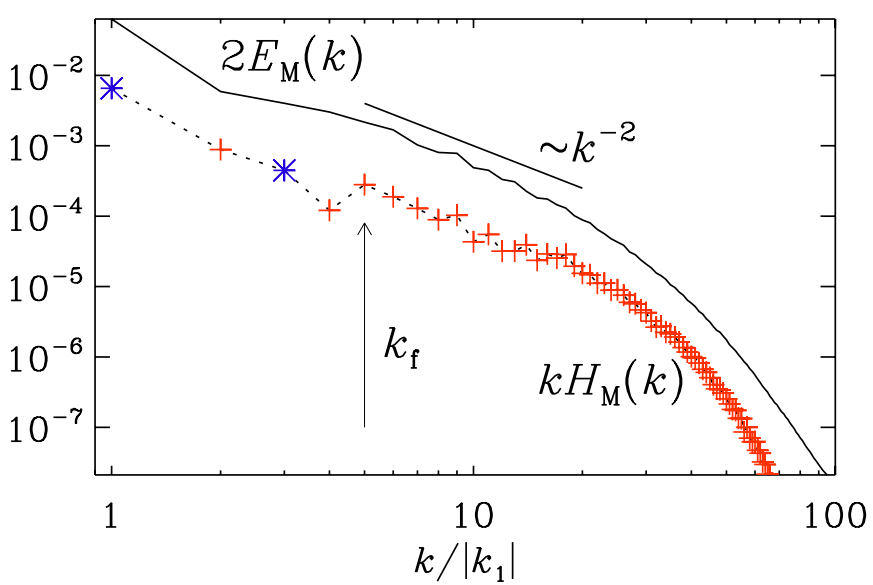

Figure 12. Three-dimensional magnetic energy and rescaled helicity spectra for the snapshot analyzed in Figure 11. The red plus signs indicate positive helicity and the blue asterisks negative helicity.

(A color version of this figure is available in the online journal.)

Next, we consider the effect of the nonlinearity of $\phi(z)$. It results in regions in the plane of the sky where $\mathcal{R}$ is now either positive or negative. Therefore, we present the results for $\hat{\mathcal{B}}$ and $P$ by averaging over only those points where $\mathcal{R} \lambda_{1}^{2}$ is in a certain interval $(2 \pi \pm 0.6$ and $-2 \pi \pm 0.6$; which is the case for about $6 \%$ of all lines of sight); see middle and last columns of Figure 11. In those points the rms value of the mean magnetic field is about 2.5 times larger than that of the fluctuating field. The resulting spectrum still shows some of the characteristic peaks, but those corresponding to the large-scale field now occur at longer wavelengths $(-2$ or +4 for $\mathcal{R} \gtrless 0)$ and those corresponding to the small-scale field at shorter wavelengths (+7.5 or -7 for $\mathcal{R} \gtrless 0$ ). Thus, the overall result is much less clear than in the idealized model, but some basic features of a bi-helical field can still be identified.

In Figure 12 we show the three-dimensional magnetic energy and helicity spectra, $E_{M}(k)$ and $H_{M}(k)$, respectively. These spectra are normalized such that $\int E_{M}(k) d k=(1 / 2)\left\langle\boldsymbol{B}^{2}\right\rangle$ and $\int H_{M}(k) d k=\langle\boldsymbol{A} \cdot \boldsymbol{B}\rangle$. The relative magnetic helicity is defined as $r_{M}=k H_{M}(k) / 2 E_{M}(k)$, whose modulus is between -1 and +1 (Moffatt 1978; Brandenburg \& Subramanian 2005). As expected, the field is bi-helical with negative magnetic helicity at $k=\left|k_{1}\right|$ and a positive one at $k=k_{\mathrm{f}}=5\left|k_{1}\right|$, but $r_{M}$ is only about $\mp 0.1$, respectively. Contributions from $k>k_{\mathrm{f}}$ are not expected to be important because of the rapid decline of spectral power proportional to $k^{-2}$. However, unlike the case without shear (Figure 1(b) of Brandenburg et al. 2012), there is no clear separation of scales and the local peak at $k=k_{\mathrm{f}}$ is barely noticeable.

Based on the results of this section, we can conclude that the reason for the departure of $\left|P\left(\lambda^{2}\right)\right|$ from the ideal case is partly the low degree of relative magnetic helicity. However, another important reason is the occurrence of a polarization peak at zero wavelength. It can interfere with the other peaks and thereby contaminate the polarization signal also at other wavelengths.

\section{CONCLUSIONS}

Our present investigations have shown that a helical magnetic field with a suitable sign of helicity can compensate Faraday depolarization and shift the polarized emission into the observable range. In practice, the magnetic field has contributions from a superposition of magnetic fields with different wavenumbers and helicities. For bi-helical magnetic fields, the bulk of the polarized emission is shifted to wavelengths whose value depends on the average wavenumber of the magnetic field. Thus, even though one of the two constituents in isolation might not be detectable (see, e.g., the top right panel of Figure 7), it could become observable because the signature of its presence would have been carried into the observable range (rows 3-5 on the right of Figure 7). However, it is equally well possible that most of the polarized emission would have been shifted out of the observable range (lower panels on the left of Figure 7). In that case, very little polarized emission can be expected.

When a galaxy is viewed edge-on, one can expect that its toroidal magnetic field can provide the line-of-sight component needed to detect helicity of field vectors in the perpendicular components. Dynamo theory predicts that this toroidal field has the same orientation above and below the midplane (Beck et al. 1996). However, the magnetic helicities of both large-scale and small-scale fields would change sign about the equatorial plane. Thus, it is conceivable that signatures of bi-helical magnetic fields would be detectable on only one of the two sides around the midplane for a fixed direction of $B_{\|}$. For edge-on galaxies, this would correspond to two opposite quadrants of detectability in the projection on the sky.

Radio emission at long (short) wavelengths would give information about magnetic fields with large (small) wavenumbers, corresponding to small (large) length scales. In galaxies, the typical scales of large-scale and small-scale magnetic fields are $1 \mathrm{kpc}$ and $\lesssim 0.1 \mathrm{kpc}$, respectively. The corresponding wavenumbers are $6 \mathrm{kpc}^{-1}$ and $\gtrsim 60 \mathrm{kpc}^{-1}$, respectively. With the numbers given at the end of Section 2, the corresponding radio wavelengths would be $\lambda_{1}=30 \mathrm{~cm}$ for the large-scale field and $\lambda_{2} \gtrsim 1 \mathrm{~m}$ for the small-scale field; see Horellou \& Fletcher (2014) for more detailed estimates. However, to resolve $P\left(\lambda^{2}\right)$ sufficiently well, it is necessary to sample both shorter and longer wavelengths. With the Square Kilometre Array, we expect to obtain polarization measurements in the range from $2 \mathrm{~cm}$ to $6 \mathrm{~m}$. With our estimate of $\lambda_{1}=30 \mathrm{~cm}$ for $k_{1}=6 \mathrm{kpc}^{-1}$, this would allow access to $\lambda^{2} / \lambda_{1}^{2}$ from 0.004 to 400 , corresponding to $k$ from $0.03 \mathrm{kpc}^{-1}$ to $2400 \mathrm{kpc}^{-1}\left(=2.4 \mathrm{pc}^{-1}\right)$ and thus spatial scales between $240 \mathrm{kpc}$ and $3 \mathrm{pc}$. This would well be compatible with the requirements for the detection of magnetic fields with helical and bi-helical properties in external galaxies by a safe margin. On the other hand, our estimates are still quite rough and not yet based on actual turbulent dynamo simulations such as those of Gressel et al. (2008). For example, if the value of $n_{e} B_{\|}$was smaller by a factor of 10 or more, this would easily necessitate access to the longer wavelength range. More importantly, contributions of the small-scale magnetic field to $B_{\|}$would substantially weaken the dependence of polarization on $\lambda^{2}$. Preliminary turbulence simulations suggest that this is indeed the case, although the basic features of the bi-helical magnetic field resulting from a turbulent dynamo can still be identified even then. Further studies of such more realistic models will be needed to assess the critical value of small-scale contributions that can still be tolerated in $B_{\|}$. There are also constraints from limited sensitivity and confusion of the signal due to turbulence affecting all spatial scales corresponding to radio wavelengths above $\lambda_{2}$. One might speculate that this might have a tendency of reducing the radio wavelength of the peak resulting from the small-scale magnetic field and enhancing the wavelength of the peak resulting from the large-scale field.

An alternative diagnostic for the presence and sign of helicity in the case of a continuous spectrum of scales is the 
cross-correlation analysis of Volegova \& Stepanov (2010). Surveys of polarized emission from diffuse turbulent sources in the magnetized interstellar medium could provide appropriate data. The presence of positive current helicity can be detected by observing positive RM in highly polarized regions in the sky and negative RM in weakly polarized regions. Conversely, negative magnetic helicity can be detected by observing negative RM in highly polarized regions and positive RM in weakly polarized regions. The cross-correlation coefficient between the degree of polarization and RM provides the relevant statistical diagnostics. Alternatively, polarization can be used instead of polarization degree. However, in that case a nonzero cross-correlation coefficient would be harder to distinguish.

Other possible targets where one can search for helical magnetic fields include the ejecta from active galactic nuclei, where evidence for swirling magnetic fields has been presented recently (Reichstein \& Gabuzda 2012), and supernova remnants, which can accelerate cosmic-ray protons across the shock, leading to a current with a component parallel to the magnetic field, which drives current helicity and an $\alpha$ effect (Rogachevskii et al. 2012). The typical radio wavelengths associated with helical magnetic fields can be estimated based on their estimated Faraday depths. For the supernova remnant G296.5+10.0, HarveySmith et al. (2010) found regions with $\mathrm{RM}=-14 \mathrm{rad} \mathrm{m}^{-2}$ and $28 \mathrm{rad} \mathrm{m}^{-2}$, corresponding to $\lambda=\sqrt{N / 2 \pi \mathrm{RM}} \approx 8-10 \mathrm{~cm}$, where we have assumed $N=2$ for the number of nodes in the slab. However, RM can show large variations, and values of $130 \mathrm{rad} \mathrm{m}^{-2}$ have been suggested for G152.4-2.1 (Foster et al. 2013 ), which would correspond to $\lambda=3.4 \mathrm{~cm}$. This would still be within the limits of what is feasible with present and future facilities.

We thank Oliver Gressel for organizing the Nordita meeting on Galactic Magnetism in the Era of LOFAR and SKA for providing an inspiring atmosphere, where the present work was started. We also acknowledge discussions with Cathy Horellou and Andrew Fletcher and thank them for sharing their related results with us. We thank Rainer Beck, Matthias Rheinhardt, Anvar Shukurov, Kandaswamy Subramanian, and the referee for useful comments and detailed suggestions that have led to improvements of the manuscript. Financial support from the European Research Council under the AstroDyn Research Project 227952, the Swedish Research Council under the grants 621-2011-5076 and 2012-5797, as well as the Research Council of Norway under the FRINATEK grant 231444 are gratefully acknowledged. R.S. acknowledges support from grant YD-520.2013.2 of the Council of the President of the Russian Federation and benefitted from the International Research Group Program supported by the Perm region government. We acknowledge the allocation of computing resources provided by the Swedish National Allocations Committee at the Center for Parallel Computers at the Royal Institute of Technology in Stockholm and the National Supercomputer Centers in Linköping, the High Performance Computing Center North in Umeå, the Nordic High Performance Computing Center in Reykjavik, and the supercomputer URAN of the Institute of Mathematics and Mechanics UrB RAS.

\section{APPENDIX}

\section{CONCERNING EQUATIONS (1)-(3)}

The purpose of this appendix is to clarify alternative definitions of Equations (1)-(3) in the literature. They are related to the position of the observer, the direction of the line-of-sight magnetic field, and the sign of the Faraday depth. We discuss three variants, referred to as I, II, and III. A commonly adopted variant is to place the observer at $z \rightarrow \infty$ and write Equation (2) as (e.g., Donner \& Brandenburg 1990; Brandenburg et al. 1993; Sokoloff et al. 1998)

$$
P\left(\lambda^{2}\right)=p_{0} \int_{-\infty}^{\infty} \epsilon(z) e^{2 \mathrm{i}\left(\psi(z)+\phi(z) \lambda^{2}\right)} d z \quad \text { (variant I). }
$$

Another convenient variant is to place the observer at $z=0$ and write Equation (2) instead as

$$
P\left(\lambda^{2}\right)=p_{0} \int_{0}^{\infty} \epsilon(z) e^{2 \mathrm{i}\left(\psi(z)+\phi(z) \lambda^{2}\right)} d z \quad \text { (variants II and III). }
$$

A second more crucial point concerns definition of the Faraday depth $\phi(z)$. For variant I (e.g., Donner \& Brandenburg 1990; Brandenburg et al. 1993; Sokoloff et al. 1998), the choice is obvious:

$$
\phi(z)=K \int_{z}^{\infty} n_{e}(s) B_{\|}(s) d s \quad(\text { variant I). }
$$

However, when the observer is at $z=0$, one can define

$$
\phi(z)=K \int_{0}^{z} n_{e}(s) \boldsymbol{B} \cdot \boldsymbol{k} d s \quad(\text { variants II and III), }
$$

where $\boldsymbol{k}$ is a unit vector pointing either toward the source (Burn 1966) or toward the observer (Frick et al. 2001). Thus, we have either (Burn 1966; Frick et al. 2010, 2011)

$$
\phi(z)=K \int_{0}^{z} n_{e}(s) B_{\|}(s) d s \quad(\text { variant II })
$$

or, as in the present paper and in many others (Frick et al. 2001; Brentjens \& de Bruyn 2005; Heald et al. 2009),

$$
\begin{aligned}
\phi(z) & =K \int_{z}^{0} n_{e}(s) B_{\|}(s) d s \\
& =-K \int_{0}^{z} n_{e}(s) B_{\|}(s) d s \quad \text { (variant III). }
\end{aligned}
$$

This formulation is also equivalent to the now-common notation where one writes (e.g., Heald 2009; Braun et al. 2010; Gießübel et al. 2013)

$$
\phi(z)=K \int_{\text {source }}^{\text {observer }} n_{e} \boldsymbol{B} \cdot d \boldsymbol{l} \quad(\text { variant III), }
$$

because $\boldsymbol{B} \cdot d \boldsymbol{l}$ is the same as our $B_{\|}(s) d s$, while source and observer correspond to $z$ and 0 , so the integral goes from $z$ to 0 .

Concerning the definition of $\phi(z)$, we emphasize that Faraday rotation of the polarization plane is a physical process that does not depend on the coordinate system or the position of the observer. Apparently, the sense of clockwise or counterclockwise rotation depends on the position of the observer with respect to the polarization plane. Consider two observers, Observer A at $z=0$ looking in the direction of $+\infty$ and Observer B at $z=+\infty$ looking toward $z=0$. The Faraday rotation corresponds then to an increase (decrease) of the polarization angle in the $(x, y)$ 
plane with increasing (decreasing) z, i.e., for a wave approaching Observer B (Observer A). However, both observers will see counterclockwise rotation of the polarization plane of the waves. A common convention is that positive RM means that the line-of-sight magnetic field between the source and the observer points toward the observer. This is the case for Equation (A3) and Equation (A6) with $\mathrm{RM}=d \chi / d \lambda^{2}$. On the other hand, with Equation (A5) one would need to write $\mathrm{RM}=-d \chi / d \lambda^{2}$, which is mathematically correct, but not recommended in view of RM synthesis techniques where Faraday depth is used with the same convention as RM. We conclude therefore that the only meaningful definitions are either Equation (A1) with Equation (A3) (variant I) or Equation (A2) with Equation (A6) (variant III).

\section{REFERENCES}

Arshakian, T. G., \& Beck, R. 2011, MNRAS, 418, 2336

Beck, R., Brandenburg, A., Moss, D., Shukurov, A., \& Sokoloff, D. 1996, ARA\&A, 34, 155

Beck, R., Fletcher, A., Shukurov, A., et al. 2005, A\&A, 444, 739

Beck, R., Frick, P., Stepanov, R., \& Sokoloff, D. 2012, A\&A, 543, A113

Beck, R., \& Wielebinski, R. 2013, in Planets, Stars and Stellar Systems, ed. T. D. Oswalt \& G. Gilmore (Dordrecht: Springer), 641

Bell, M. R., \& Enßlin, T. A. 2012, A\&A, 540, A80

Blackman, E. G., \& Brandenburg, A. 2002, ApJ, 579, 359

Brandenburg, A. 2001, ApJ, 550, 824

Brandenburg, A., Candelaresi, S., \& Chatterjee, P. 2009, MNRAS, 398, 1414

Brandenburg, A., Donner, K. J., Moss, D., et al. 1993, A\&A, 271, 36

Brandenburg, A., Sokoloff, D., \& Subramanian, K. 2012, SSRv, 169, 123

Brandenburg, A., \& Subramanian, K. 2005, PhR, 417, 1

Brandenburg, A., Subramanian, K., Balogh, A., \& Goldstein, M. L. 2011, ApJ, 734, 9

Braun, R., Heald, G., \& Beck, R. 2010, A\&A, 514, A42

Brentjens, M. A., \& de Bruyn, A. G. 2005, A\&A, 441, 1217

Burn, B. J. 1966, MNRAS, 133, 67

Donner, K. J., \& Brandenburg, A. 1990, A\&A, 240, 289
Elstner, D., Meinel, R., \& Beck, R. 1992, A\&AS, 94, 587

Field, G. B., \& Blackman, E. G. 2002, ApJ, 572, 685

Fletcher, A. 2010, in ASP Conf. 438, The Dynamic Interstellar Medium, ed. R. Kothes et al. (San Francisco, CA: ASP), 197

Foster, T. J., Cooper, B., Reich, W., Kothes, R., \& West, J. 2013, A\&A, 549, A107

Frick, P., Sokoloff, D., Stepanov, R., \& Beck, R. 2010, MNRAS, 401, L24

Frick, P., Sokoloff, D., Stepanov, R., \& Beck, R. 2011, MNRAS, 414, 2540

Frick, P., Stepanov, R., Shukurov, A., \& Sokoloff, D. D. 2001, MNRAS, 325,649

Gießübel, R., Heald, G., Beck, R., \& Arshakian, T. G. 2013, A\&A, 559, A27

Ginzburg, V. L., \& Syrovatskii, S. I. 1965, ARA\&A, 3, 297

Gressel, O., Elstner, D., Ziegler, U., \& Rüdiger, G. 2008, A\&A, 486, L35

Harvey-Smith, L., Gaensler, B. M., Kothes, R., et al. 2010, ApJ, 712, 1157

Heald, G. 2009, in IAU Symp. 259, Cosmic Magnetic Fields: From Planets, to Stars and Galaxies, ed. K. G. Strassmeier et al. (Cambridge: Cambridge Univ. Press), 591

Heald, G., Braun, R., \& Edmonds, R. 2009, A\&A, 503, 409

Horellou, C., \& Fletcher, A. 2014, MNRAS, in press (arXiv:1401.4152)

Junklewitz, H., \& Enßlin, T. A. 2011, A\&A, 530, A88

Käpylä, P. J., \& Brandenburg, A. 2009, ApJ, 699, 1059

Kleeorin, N., Moss, D., Rogachevskii, I., \& Sokoloff, D. 2000, A\&A, 361, L5

Moffatt, H. K. 1978, Magnetic Field Generation in Electrically Conducting Fluids (Cambridge: Cambridge Univ. Press)

Oppermann, N., Junklewitz, H., Robbers, G., \& Enßlin, T. A. 2011, A\&A, 530, A89

Pacholczyk, A. G. 1970, Radio Astrophysics (San Francisco: Freeman)

Reichstein, A., \& Gabuzda, D. 2012, JPhCS, 355, 012021

Rogachevskii, I., Kleeorin, N., Brandenburg, A., \& Eichler, D. 2012, ApJ, 753, 6

Sofue, Y., Fujimoto, M., \& Wielebinski, R. 1986, ARA\&A, 24, 459

Soida, M., Krause, M., Dettmar, R.-J., \& Urbanik, M. 2011, A\&A, 531, A127

Sokoloff, D. D., Bykov, A. A., Shukurov, A., et al. 1998, MNRAS, 299, 189

Taylor, J. H., \& Cordes, J. M. 1993, ApJ, 411, 674

Urbanik, M., Elstner, D., \& Beck, R. 1997, A\&A, 326, 465

Volegova, A. A., \& Stepanov, R. A. 2010, JETP Lett., 90, 637

Waelkens, A. H., Schekochihin, A. A., \& Enßlin, T. A. 2009, MNRAS, 398, 1970

Woltjer, L. 1958, PNAS, 44, 489

Zhang, H., Brandenburg, A., \& Sokoloff, D. D. 2014, ApJL, 784, L45 